\title{
Network Reconstruction Reveals that Valproic Acid Activates Neurogenic Transcriptional Programs in Adult Brain Following Traumatic Injury
}

\author{
Gerald A. Higgins' - Patrick Georgoff ${ }^{2}$ - Vahagn Nikolian ${ }^{2}$ - Ari Allyn-Feuer' ${ }^{\prime}$ Brian Pauls $^{3}$. \\ Richard Higgins ${ }^{4}$ Brian D. Athey ${ }^{1,5} \cdot$ Hasan E. Alam²
}

Received: 7 November 2016 / Accepted: 17 February 2017 / Published online: 7 March 2017

(C) The Author(s) 2017. This article is published with open access at SpringerLink.com

\begin{abstract}
Objectives To determine the mechanism of action of valproic acid (VPA) in the adult central nervous system (CNS) following traumatic brain injury (TBI) and hemorrhagic shock (HS). Methods Data were analyzed from different sources, including experiments in a porcine model, data from postmortem human brain, published studies, public and commercial databases.

Results The transcriptional program in the CNS following TBI, HS, and VPA treatment includes activation of regulatory pathways that enhance neurogenesis and suppress gliogenesis. Genes which encode the transcription factors (TFs) that specify neuronal cell fate, including MEF2D, MYTIL, NEURODI, PAX6 and TBRI, and their target genes, are induced by VPA. VPA represses genes responsible for oligodendrogenesis, maintenance of white matter, T-cell activation, angiogenesis, and endothelial cell proliferation, adhesion and chemotaxis. NEUROD1
\end{abstract}

has regulatory interactions with $38 \%$ of the genes regulated by VPA in a swine model of TBI and HS in adult brain. Hi-C spatial mapping of a VPA pharmacogenomic SNP in the GRIN2B gene shows it is part of a transcriptional hub that contacts 12 genes that mediate chromatinmediated neurogenesis and neuroplasticity.

Conclusions Following TBI and HS, this study shows that VPA administration acts in the adult brain through differential activation of TFs responsible for neurogenesis, genes responsible for neuroplasticity, and repression of TFs that specify oligodendrocyte cell fate, endothelial cell chemotaxis and angiogenesis.

Short title: Mechanism of action of valproic acid in traumatic brain injury

KEY WORDS bioinformatics · network modeling ·

pharmacoepigenomics

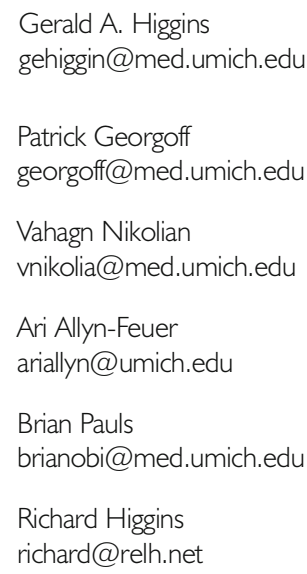

Brian D. Athey

bleu@med.umich.edu

Hasan E. Alam

alamh@med.umich.edu

Department of Computational Medicine and Bioinformatics, University of Michigan Medical School, Ann Arbor, Michigan, USA

2 Department of Surgery, University of Michigan Medical School, Ann Arbor, Michigan, USA

3 College of Pharmacy, University of Michigan, Ann Arbor, Michigan, USA

4 Department of Computer Science, University of Maryland, College Park, Maryland, USA

5 Michigan Institute for Data Science (MIDAS), Ann Arbor, Michigan, USA 


\section{ABBREVIATIONS}

ChIP-seq ChIP-sequencing is a method used to analyze protein interactions with DNA

eQTL Expression quantitative trait loci

GWAS Genomewide association studies

hQTL Histone quantitative trait locus

H3 Histone 3 , one of the 5 main histone proteins involved in the structure of the nucleosome in the chromatin of eukaryotic cells

H3K9me2/ Histone H3 di- or tri-methylated at residue lysine

39 . histone marks that are found in facultatively repressed genes

H3K27ac Histone H3 acetylated at residue lysine 27. $\mathrm{H} 3 \mathrm{~K} 27 \mathrm{ac}$ is associated with the higher activation of transcription and therefore defined as an active mark of both enhancers and promoters

H3K27me2/ Histone H3 di- or tri-methylated at residue lysine

327 . histone marks that are found in facultatively repressed genes

H4 Histone 4, one of the 5 main histone proteins involved in the structure of the nucleosome in the chromatin of eukaryotic cells

HDAC Histone deacetylase

HOT High occupancy by transcription factors. indicative of enhancers and promoters, especially in developmentally important genes

HS Hemorrhagic shock

$\mathrm{IC}_{50} \quad$ Concentration of an inhibitor where the response (or binding) is reduced by half

TAD Topologically-associating domain, the basic spatial unit of transcription in the human genome

TBI Traumatic brain injury

TF Transcription factor

SK-N-SH SK human caucasian neuroblastoma, an immortalized human neuronal cell line

VPA Valproic acid

\section{GENE SYMBOLS}

ARIDIA

ASCLI

ATF7IP

$B C L|| A$

BCL2L I |

BMP2

BTG2

CASP3

CCDCCII7

CCL2

$\mathrm{CHAFIB}$

CTCF

CYR6I
AT-rich interaction domain 1A

Achaete-Scute family BHLH transcription

factor 1

Activating transcription factor 7 interacting protein

B-Cell CLL/Lymphoma 11A

BCL2 like 11

Bone morphogenetic protein 2

BTG family member 2

Caspase 3

Coiled-coil domain containing 117

Chemokine C-C Motif Ligand 2

Chromatin assembly factor 1 subunit B

CCCTC-binding factor

Cysteine rich angiogenic inducer
ELKI

EPHB2

EWSRI

FGF7

FGF9

GRASP

GRIN2B

ILIRN

KREMENI

MEF2C

MEF2D

MYTIL

NEURODI

NFI

NRIDI

NR4AI

NR6AI

OPALIN

PAX6

PMP2

PPARD

PROXI

PRRXI

SETDBI

SIRTI

SMARCB I

$50 \times 10$

STI 8

TBRI

THBSI

$\mathrm{XBP} I$

ZNRF3
ELK1 ETS transcription factor

EPH receptor $\mathrm{B} 2$

EWS RNA binding protein 1

Fibroblast growth factor 7

Fibroblast growth factor 9

GRP1 (General receptor for

phosphoinositides 1)-associated scaffold

protein

Glutamate ionotropic receptor NMDA type subunit 2B

Interleukin 1 receptor antagonist

Kringle Containing Transmembrane Protein 1

Myocyte enhancer factor 2C

Myocyte enhancer factor 2D

Myelin transcription factor 1 Like

Neuronal differentiation 1

Neurofibromin 1

Nuclear receptor subfamily 1 group D member 1

Nuclear receptor subfamily 4 group A member 1

Nuclear receptor subfamily 6 group A member 1

Oligodendrocytic Myelin Paranodal and Inner Loop Protein

Paired box 6

Peripheral Myelin Protein 2

Peroxisome proliferator activated receptor delta

Prospero homeobox 1

Paired related homeobox 1

SET domain bifurcated 1

Sirtuin 1

SWI/SNF related matrix associated, actin dependent regulator of chromatin, subfamily B, member 1

SRY-box 10

Suppression of Tumorigenicity 18 zinc finger

T-box brain 1

Thrombospondin 1

X-box binding protein 1

Zinc and ring finger 3

\section{INTRODUCTION}

The mechanism of action of VPA as an anticonvulsant, mood stabilizer and analgesic in the human central nervous system (CNS) has not been adequately characterized. VPA and its derivatives exhibit a variety of effects which are cell- and tissue-specific, differ based on disease state, age, gender and ethnicity, and may be effective or deleterious. VPA is a weak 
blocker of sodium and calcium ion channels, and may inhibit key enzymes in the catabolism of gamma-aminobutyric acid (GABA), including ABAT (4-aminobutyrate aminotransferase) at physiologically relevant concentrations (1). There is also evidence that VPA exerts its antiepileptic action through differential regulation of the GRIN2B (Glutamate Ionotropic Receptor NMDA Type Subunit 2B) gene (2). VPA is an effective inhibitor of histone deacetylases (HDACs), with an $\mathrm{IC}_{50}(0.4 \mathrm{mM})$ well within the therapeutic range of VPA $(0.35-0.7 \mathrm{mM}$ in serum). VPA causes robust chromatin decondensation, with potent acetylation of core histones such as $\mathrm{H} 3$ and $\mathrm{H} 4$ that leads to activation of development gene expression $(3,4)$. VPA has been shown to be neuroprotective in animal models of traumatic brain injury (TBI) (5,6), spinal cord injury (7), and in neurodegenerative disease (8). In a swine model of TBI and hemorrhagic shock (HS) VPA decreases brain lesion size, improves neurologic recovery, and down-regulates genes associated with necrosis, apoptosis, and inflammation (9). The HDAC inhibitor, sodium butyrate, with a mechanism of action like that of VPA, has been shown to activate neurogenesis in rodent brain following ischemic injury (10). These results suggest different ways in which VPA may act in parallel to provide benefit in epilepsy, mood disorders, migraine, as well as recovery following trauma.

It appears that VPA-induced histone acetylation is not sufficient for chromatin decondensation, but rather a downstream effect of HDAC inhibition, suggesting that the drug suppresses the expression of proteins involved in maintenance of heterochromatin and/or uses chromatin remodeling proteins as intermediaries (11-13). It is also routinely used to remove histone methylation in cellular domains such as the lamina-associating domain (LAD) located just interior of the nuclear membrane, a region containing heterochromatin, in which exposure to VPA abolishes H3K27 and H3K9 methylation (14).

Recent studies of the epigenomic control of gene expression that has identified distinct mechanisms through which chromatin interactions mediate transcriptional programs involving topologically-associating domains (TADs), enhancerpromoter loops and actively transcribed regions of the human genome characterized by the histone mark H3K27ac (15-17). It has been shown that SNPs which disrupt the boundaries of TADs cause serious health problems (18).; and causal SNPs exhibit significant allele bias in open chromatin (19). In addition, the greater the number of spatial connections a given enhancer or promoter maintains genomewide indicates both the potency of the regulatory element and its validity (20), especially for CNS genes that are involved in coordinated transcriptional programs of neurogenesis and neuroplasticity (20).

Our hypothesis is that VPA acts through transcriptional activation and repression of specific genes resulting in chromatin-mediated neurogenesis and neuroplasticity in the adult CNS and inhibition of glial scarring. To determine the validity of this premise, we analyzed experimental and public data. We then reconstructed a gene regulatory network that mediates VPA's mechanism of action in human brain, and found that the drug exerts widespread effects in adult brain including a transcriptional program of neurogenesis and neuroplasticity, involving TFs that have been shown to program neuronal cell fate commitment and suppress oligodendrocyte cell fate.

\section{METHODS}

\section{Data}

Figure 1 shows an overview of the experimental design. Public databases and sources used in this analysis are specified in Supplementary Table 1 . These included experimental results from a swine model of TBI and hemorrhage $(5,9)$. Other primary data included microarray expression data from postmortem human brain tissue obtained from the Human Brain Atlas of the Allen Brain Science Institute (21) and other sources (22).

\section{Experimental data from an animal model of TBI and HS}

Examination of gene expression data from a swine model of TBI and Hemorrhagic Shock exhibited functional recovery following VPA therapy showed remarkable enrichment of transcription factors ( $\mathrm{TFs}$ ) that were regulated in a significant manner by the drug. In the animal model, swine were subjected to controlled TBI and hemorrhage $(40 \%$ blood volume), kept in shock for $2 \mathrm{~h}$, and resuscitated with vehicle or vehicle + VPA ( $n=3$ per group). The vehicle was Hextend (Hospira Inc. Lake Forest, IL) at $50 \mathrm{~mL} / \mathrm{min}$ versus (2) Hextend at $50 \mathrm{~mL} / \mathrm{min}$ plus VPA at $300 \mathrm{mg} / \mathrm{kg}$ (EMD Biosciences Inc., La Jolla, CA). In the Hextend + VPA group, VPA treatment was started $1 \mathrm{~h}$ after hemorrhage at an infusion rate of $100 \mathrm{mg} / \mathrm{kg}$ per hour intravenously. The vehicle and treatment groups received identical volumes of fluid, which matched the volume of shed blood. After $6 \mathrm{~h}$, brain RNA was isolated, and gene expression profiles were measured using a Porcine Gene ST 1.1 microarray (Affymetrix, Santa Clara, CA).

\section{Gene expression data from postmortem human brain}

Results of microarray expression analysis from postmortem human brain were obtained from different sources $(21,22)$. In a patient cohort with epilepsy, patients on VPA therapy were compared to controls at time of death (TOD) for relative levels of several TFs, nuclear receptors, and other mRNAs (23). Sample sizes for each group were $\mathcal{N}=6$ for epileptic 
Fig. I Overview of the experimental design used in this study. (I) Shows steps to annotate putative VPA pharmacogenomics SNPS which were (2) mechanistically analyzed using integrative bioinformatics and regulatory network analysis methods. In (I) numbers refer to the different annotation steps of the bioinformatics pipeline. Details can be found in the text and in Supplement.
(1) Annotation of pharmacogenomic SNPs that act in human brain in central VPA pathways

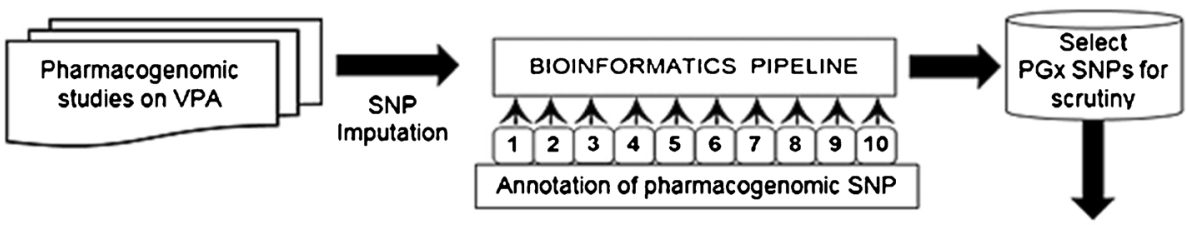

(2) Reconstruction of gene regulatory networks to determine VPA's mechanism of action in human CNS

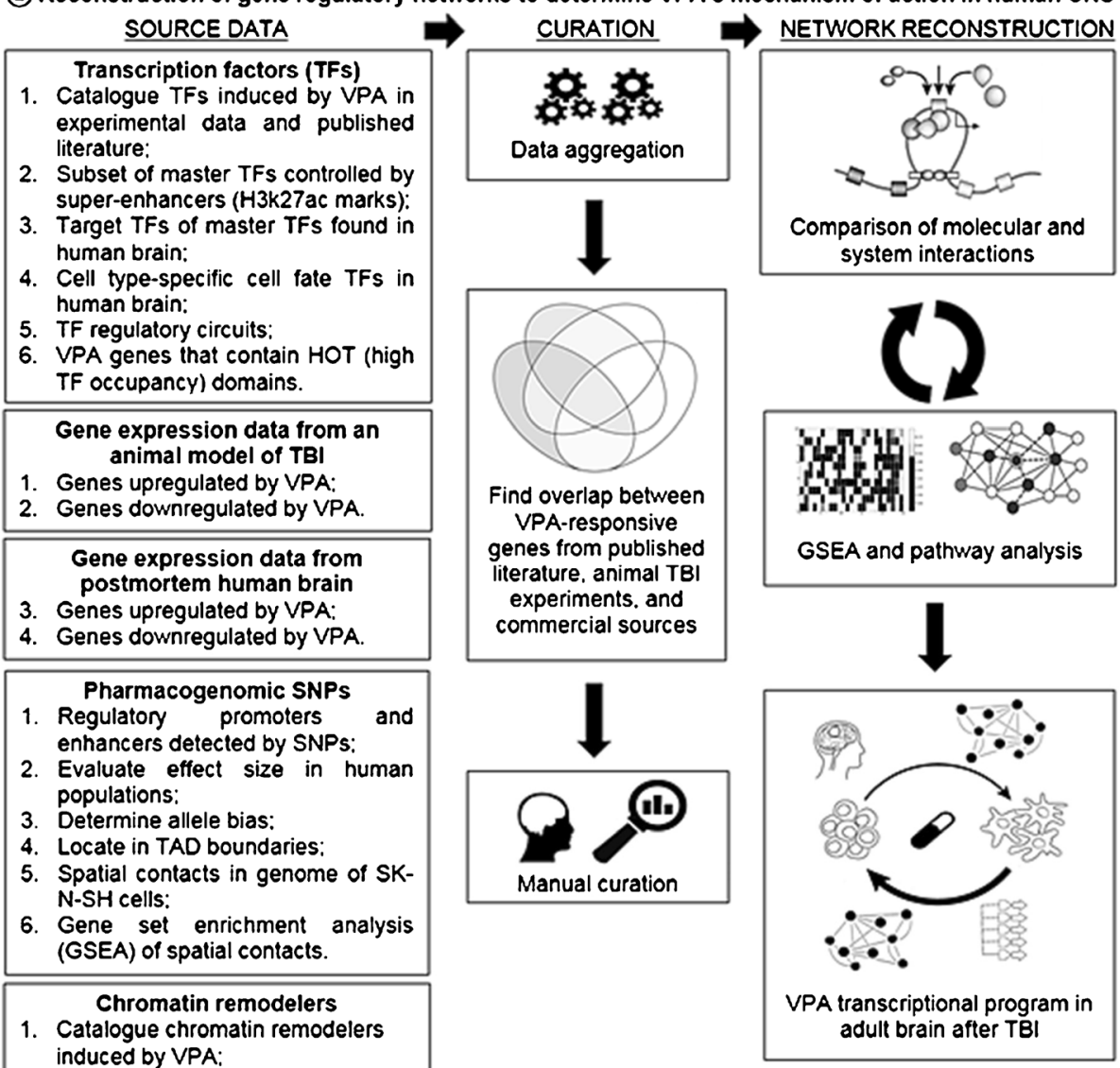

patients on VPA therapy at TOD, $\mathcal{N}=6$ for epileptic patients on a different anti-convulsant drug at TOD.

\section{Experimental data from the human SK-N-SH cell line}

Publicly available date from SK-N-SH cells were used for two applications: (1) For determination of DNAse I hypersensivity and allele bias, as they are included as examples in the deltaSVM machine learning algorithm (19), and (2) Evaluation of spatial interactions in the human genome using data from a high resolution Hi-C dataset (19). To discover how the VPA pharmacogenomic SNPs we selected produced robust pharmacogenomic stratification, evaluation of the chromatin interactions of regulatory elements they were located in was used to preliminarily map the putative VPA pharmacodynamic pathway in human brain.
SK-N-SH cells are a human neuroblastoma cell line which $>$ contains a mixture of at least 2 cell types.

\section{Published literature on pharmacogenomic associations and VPA targets and pathways}

Two hundred and fifty-four peer-reviewed published articles were retrieved between January 1 and October 1 2016, including gene association studies, as well as basic and clinical pharmacology reports. An automated Boolean search string was used in PubMed (24), Google Scholar (23) and clinicaltrials.gov (25), consisting of "valproate OR valproic acid OR sodium valproate OR divalproex sodium AND pharmacodynamics OR mechanism of action OR brain OR pathways AND pharmacogenomic AND bipolar disorder AND epilepsy AND migraine headache AND gene OR 
SNP AND association AND human." There are no published genomewide association studies (GWAS) that are focused on medication response or adverse events related to only valproic acid therapy in epilepsy, bipolar disorder or migraine. There are however, several GWAS that have investigated SNPs associated with human populations that have treatmentresistant or refractory epilepsy. These studies are made up primarily of patients who do not respond to a combination of anticonvulsant drugs or worsen on therapeutic regimens. Due to confounding related to polypharmacy these studies were excluded. For this study, we avoided anything but primary research studies in which we re-tested all the parameters and statistical tests that were employed. This dramatically reduced the number of studies from 254 to 52 that could be included in this analysis.

\section{Analysis}

\section{VPA pharmacogenomic SNPs}

To better understand human CNS pathways that are involved in the mechanism of action of valproic acid, we combined SNP imputation with bioinformatics analysis as performed in a previous study (22). SNPs included in the analysis were those likely to be associated with known biological targets of VPA, could be functionally annotated, and were derived from primary research studies. For this analysis of potential pharmacodynamic pathways, we excluded pharmacokinetic genes and their variants such as the $U G T$ and $C Y P$ super-families. To select VPA pharmacogenomic SNPs we combined SNP imputation from VPA pharmacogenomic association studies, and performed computational and bioinformatics analysis as described in detail (22). DrugBank (25) and the pharmacogenomic mutation database (PGMD®; Qiagen) lists pharmacodynamic targets that have been associated with VPA (26), and these were also interrogated to gather additional information.

In Fig. 1.1, bioinformatics analysis was used to identify the most probable causal SNP from the association regardless of the lead SNP(s) that were reported, and included the following bioinformatics analysis to determine the functionality of the variant (22): (1) Location in open chromatin as indicated by peaks of DNAse I hypersensitivity, (2) Low to moderate methylation of any cytosine residues, (3) The presence of histone marks that indicate regulatory function $(\mathrm{H} 3 \mathrm{~K} 27 \mathrm{ac}+$ $\mathrm{H} 3 \mathrm{~K} 4 \mathrm{me} 1$ = enhancer; H3K27ac $+\mathrm{H} 3 \mathrm{~K} 4 \mathrm{me} 3$ = promoter), (3) The location of the variant within the context of the gene or in an intergenic domain, (4) Whether the regulatory element has yet to be annotated as a molecular quantitative trait locus (eQTL, hQTL, etcetera), (4) Proteins, including transcription factors, bound to the regulatory element as determined by ChIP-Seq indicating its regulatory function, (5) Disruption by the pharmacogenomic SNP of transcription factor binding sites as indicated by alterations in the position weight matrix, (6) Association of the regulatory element with the requisite RNA species (e.g., bi-directional enhancer RNA = enhancer; mRNA = promoter), (7) Connectivity of the regulatory element with other elements in the genome as indicated by the Hi-G chromatin conformation capture method limited to SK-N-SH cells, which are the ENCODE Tier 2.5 neural surrogate cell line, (8) Transcriptional programming by factors which are responsible for determination of neuronal cell fate, (9) Determination of the allele bias of the allelic variant using the deltaSVM algorithm (19), (10) examination of the neuroanatomical distribution of target gene expression data in postmortem human brain using both microarray and in situ hybridization data from the Human Brain Atlas of the Allen Brain Science Institute (21).

\section{Harmonization of nomenclature: pig, human and rodent genes and their regulatory elements}

This study involves cross-species comparisons from pig (Sus scrofa), rat (Rattus norvegicus) and human (Homo sapiens). To ensure that we could reliably use inter-species nomenclature in the context of the human CNS, we compared gene, promoter and enhancer data using 3 resources: (1) comparative assessment of pig, rodent and human transcriptomes for identity (27), (2) thorough analysis of human accelerated regions in human brain that are not present in other mammalian brains for exclusion (28), (3) cross-species assessment of histone modifications, open chromatin and deep learning methods applied to pigs, mice, rats and humans to ensure concordance of identifiable enhancers, promoters, super-enhancers and master TFs (29), and (4) enhancer-promoter loop conservation across species using context-dependent conservation (30). All gene symbols and homologous regulatory elements were found to be the same, at least in regards to nomenclature, chromatin state annotation, and relative position within the genome. We then harmonized our nomenclature with the results of the roadmap epigenome mapping consortium. Thus, gene and protein symbols and definitions were consistent with HGNG nomenclature, except as indicated by citation in the text. Gene symbols are from GENGODE except where indicated, and genomic location coordinates are from build 38 of the Human Genome.

For annotation, we focused on active enhancers, promoters and transcription start sites, so other chromatin states were not used in the present study, including flanking and poised regulatory elements, transcribed domains or repressed elements. Although potential regulatory variants may be repressed in one tissue or brain region but not another, we could directly assess function in any biological system in this study, so we relied on the data provided by the association studies. 


\section{VPA-regulated transcription factors (TFs)}

Next, we examined data on TFs, including master TFs as defined by super-enhancer regulation in human brain that are regulated by VPA, and TFs and genes that are targets of these Master TFs (see Table I for the complete list of data sources). We examined previously characterized transcriptional networks that contained VPA-responsive TFs, results on conserved network topology from model systems, organisms and mechanisms of neurogenesis $(34,35)$ and known gene regulatory network dynamics (36-38). We also looked at the overlap of VPA genes which encode TFs and HOT (high-occupancy target) regions, which are bound by large number of transcription factors (39). Other data sources are detailed in Supplementary Table 1.

\section{Chromatin remodeling proteins and complexes}

Several and chromatin remodelers, including ARID1A, BCL11A, CHAF1B, were among genes regulated by VPA. We determined the regulatory targets of these genomewide, and determined and to what degree they overlapped the VPA-responsive promoters as part of the reconstruction of the network. We also examined pioneer factors such as PAX6, which appears to act in concert with chromatin remodeling complexes during neurogenesis, including published data on TFs that are pioneer factors and chromatin state regulators (Supplementary Table 1).

\section{Reconstruction of VPA gene regulatory networks in the human CNS}

Figure 1.2 shows the method by which we reconstructed the central VPA pathway in the human CNS. It involved: Identification of databases relevant to modeling the VPA regulatory circuit, Data aggregation from public databases and in-house data, manual curation of the data in the context of a VPA central mechanism of action, preparation of curated data for model integration, iterative gene set enrichment analysis (GSEA) using different public and commercial software, and pathway analysis using IPA ${ }^{\circledR}$ and the STRING database of protein-protein interactions (40), as well as network analysis using weighted gene co-expression network analysis (WGCNA) in R (41) and node-edge modeling systems modeling using Python (42). In addition to applying redundant software analysis tools, we also used different open source and commercial databases, including IPA® (43), Pathway Commons (44) and Reactome (45) databases as well as manual curation of the scientific literature, to determine network interactions.

For reconstruction of VPA's gene CNS regulatory pathway, we used a hybrid model development method that combines GSEA and pathway analysis/network modeling software used in bioinformatics with a technique for constructing core regulatory circuitry which includes super-enhancers, TFs and auto-regulation, based on defined biological attributes of transcriptional regulation in the human CNS (Supplementary Table 1). During the project, we discovered several other TFs that are regulated by VPA that directs cell fate, in addition to many genes whose was expression was regulated by ASCL1 (Achaete-Scute Family BHLH Transcription Factor 1), NEUROD1 (Neuronal Differentiation 1), MEF2C (Myocyte Enhancer Factor 2C), MEF2D (Myocyte Enhancer Factor 2D), MYT1L (Myelin Transcription Factor 1 Like), and TBR1 (T-Box, Brain 1). Many TFs were tightly coupled in terms of target promoters, many of which were also involved in neurogenesis and neuroplasticity. As such, this convoluted super-program was investigated in greater detailed.

\section{Relationship between VPA-regulated TFs and other TFs in human brain and cell types}

To understand the relationship between genes whose expression is highly regulated by VPA following brain injury in the CNS, we first examined sets of TFs which were known to be differentially regulated by VPA in brain and whose function was linked to neurogenesis and suppression of gliogenesis in human brain. Comparisons were made between VPA-

Table I Selected Pharmacogenomic Variants Selected for Allelic Variation in VPA Dose, Response and Pharmacodynamics in Human Populations. Noncoding SNPs that Identified Regulatory Elements, Including Enhancers and Promoters

\begin{tabular}{|c|c|c|c|c|c|c|c|}
\hline SNP & GENE $^{\mathrm{a}}$ & TYPE & $\begin{array}{l}\text { deltaSVM } \\
\text { sCORE }^{\text {b }}\end{array}$ & DISEASE & EFFECT & EFFECT SIZE & REF \\
\hline rs2857654_A & CCL2 & Enhancer & -2.275704 & Epilepsy & Response in children & $1.45(1.06-1.99)$ & $(3 \mid)$ \\
\hline rs3764028_G & GRIN2B & Promoter & -5.097029 & Epilepsy & Dose range & ।.7553 (I.219-2.291) & (32) \\
\hline rs2269577_G & $X B P I$ & Promoter & 4.710044 & $\mathrm{BPD}$ & Response $^{c}$ & $1.2754(0.329-2.221)$ & (33) \\
\hline
\end{tabular}

\footnotetext{
${ }^{a}$ RefSeq nomenclature

${ }^{b}$ deltaSVM is a machine learning algorithm that determines the causal nature of gene variants, including DNAse I hypersensitivity and allele bias ( $\mathrm{I}$ )

'Total treatment response score, Kruskal-Wallis test for valproate prophylactic treatment response. BPD: Bipolar disorder, sample containing patients with BPD I and BPD 2. Effect sizes generated using Cohen's D-test adjusted for sample size
} 
responsive master TFs and their targets and other $\mathrm{TFS}_{\mathrm{s}}$ in the human telencephalon, and the different sets are represented on a coronal section of the human telencephalon (Fig. 4). Adult human brain regions, examined in the roadmap epigenome mapping consortium (15), included the angular gyrus, anterior caudate, cingulate gyrus, hippocampal formation, inferior temporal lobe and mid-frontal lobe. Cell types included astrocytes and HUVEC, the latter a lymphoblastoid cell line derived from human vascular endothelial cells.

TFs were assigned using a subset of ENCODE brain regions and in astrocytes based on annotation of super-enhancer status using histone marks such as H3K27ac and other characteristics $(15,17)$. From this analysis, only 8 of the TFs and nuclear receptors regulated by VPA exhibit enhancer-like attributes in various brain regions. Other enriched TFs include NFIX, regulated by CHAF1B and SOX2. The correlation between VPA-TFs and TFs which function with superenhancer status in different human brain regions is obvious, with this small subset accounting for $31-48 \%$ of all TFs across the telencephalon. In astrocytes, as shown in Fig. 4, although only MEF2D is represented as a VPA-TF, 17\% of all TFs in this cell type are regulated by VPA-TFs. HUVEC cells were used as a surrogate for CNS endothelial cells, and MEF2C, MEF2D and NR1D1 are VPA-TFs, with these TFs and their targets accounting for $18 \%$ of the TFs in this lymphoblastoid cell line

\section{RESULTS}

\section{VPA regulation of gene expression following injury to the brain in a swine model}

Most the genes differentially-regulated by VPA were several master TFs that are highly expressed during development of the CNS, and are also found in the developing adult human telencephalon. TFs that target the greatest number of differentially expressed genes were NEUROD1, ASCL1, MEF2C, MEF2D, BCL2L11 (BCL2 Like 11), ELK1 (ELK1, ETS Transcription Factor), MYT1L, PPARD (Peroxisome Proliferator Activated Receptor Delta), SIRT1 (Sirtuin 1), SMARCB1 (SWI/SNF Related, Matrix Associated, Actin Dependent Regulator Of Chromatin, Subfamily B, Member 1), and TF (Transferrin). All of these genes except for $S M A R C B 1$, which is induced by VPA, were found in the VPA-regulated set.

Analysis of significantly up- or -down-regulated genes in the porcine model of TBI and HS showed that NEUROD1 was involved in the regulation of $63 \%$ of the regulated genes in this dataset. Using Fisher's exact test, the $p$-value of NEURDO1-regulated genes in the dataset versus genes with those not known to be regulated by NEUROD1 is $p=1.6 \mathrm{E}-05$. Figure 2 shows the distribution of non-overlapping NEUROD1 interactions in this dataset. The most common target of overlapping TFs was CASP3 (Caspase 3), which is the target of 8 different TFs, and SIRT1, PPARD and TBR1, all targeted by 5 different TFs. Common target TFs and nuclear receptors involved in development and neuronal programming included the up-regulated genes NR6A1 (Nuclear Receptor Subfamily 6 Group A Member 1; $1.75 \log ^{2}$-fold), BCL11A (B-Cell CLL/Lymphoma 11A; $1.6 \log ^{2}$-fold), MYT1L (Myelin Transcription Factor 1 Like; $1.6 \mathrm{log}^{2}$-fold), TBR1 (1.49 $\log ^{2}$-fold), NR1D1 (Nuclear Receptor Subfamily 1 Group D Member 1, $1.42 \log ^{2}$-fold) and MEF2C (1.42 $\log ^{2}$-fold). Among VPA-responsive genes that were up-regulated, there was also significant representation of thyroxine-responsive genes, neurotransmitter genes including corticotrophin releasing hormone, and genes which encode proteins involved in neuronal cell adhesion, synaptogenesis, axonal growth, dendritic arborization and the cytoskeleton. For additional information about programmers of cell fate regulated by VPA following CNS injury, please refer to the Supplement.
Fig. 2 Types of NEUROD I control of genes differentially regulated by VPA in adult swine brain following injury. The I I enhancers that control NEUROD I include genes that are co-regulated by the same enhancer as NEURODI and genes which harbor single gene variants that impact enhancers that affect NEUROD I gene expression. For details see Supplemental dataset.

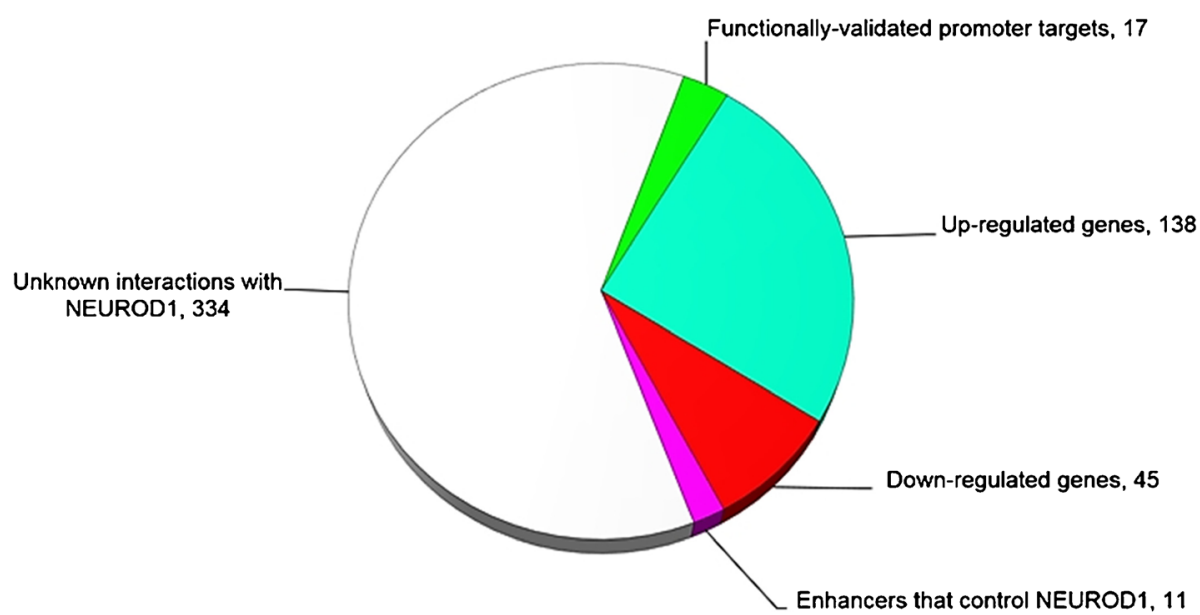


TFs that program oligodendrocytic cell fate, endothelial cell fate and angiogenesis were significantly down-regulated compared to other TFs in this dataset $(p=2.78 \mathrm{E}-04$; Fisher's exact test). These included FGF7 (Fibroblast Growth Factor 7; -3.2 $\log ^{2}$-fold), PROX1 (Prospero Homeobox 1; - $1.81 \log ^{2}$ fold), SOX10 (SRY-Box 10; -1.81 $\log ^{2}$-fold), PRRX1 (Paired Related Homeobox 1; BMP2 (Bone Morphogenetic Protein 2; $-1.73 \log ^{2}$-fold) and ST18 (Suppression Of Tumorigenicity 18, Zinc Finger; $-1.22 \log ^{2}$-fold). Other significantly downregulated genes included CRR61 (Cysteine Rich Angiogenic Inducer), OPALIN (Oligodendrocytic Myelin Paranodal And Inner Loop Protein), PMP2 (Peripheral Myelin Protein 2) and THBS1 (Thrombospondin 1).

Supplementary Figure 3 shows the significant functions of the 50 most up-regulated and most down-regulated genes following VPA therapy in a swine model of TBI and hemorrhage. These results show that in an animal model of TBI and hemorrhage, VPA therapy induces genes associated with neurogenesis and neuroplasticity, and represses those associated with cell loss, endothelial cell invasion, and angiogenesis.

\section{Selection of VPA pharmacogenomic SNPs for further examination}

Table I shows VPA pharmacogenomic SNPs that were selected for further analysis. These SNPs stratify response to VPA in human populations, and all 3 are located in regulatory domains (promoters or enhancers), and exhibit significant chro- matin allele bias as measured by the deltaSVM algorithm (19). They include:

1. The intronic SNP rs2857654_A located within the CCL2 gene, which encodes the Chemokine C-C Motif Ligand 2, and is most significantly associated with cell movement and migration of cells and this enhancer interacts with VPA (31);

2. The 5' SNP rs3764028_G located in the distal GRIN2B promoter (32). Spatial mapping in SK-N-SH cells shows that it forms an inter-chromosomal transcriptional hub (Fig. 3a), contacting genes in cis- and trans- in SK-N-SH cells that are enriched for genes involved in neuronal differentiation and development of the CNS (Fig. 3a);

3. The 5' SNP rs2269577_G located in the promoter of the XBP1 (X-Box Binding Protein 1) gene (33), which functions as a transcription factor during endoplasmic reticulum (ER) stress by regulating the unfolded protein response. Required for cardiac myogenesis and hepatogenesis during embryonic development, and the development of secretory tissues such as exocrine pancreas and salivary gland. An enhancer co-regulates XBP1, EWSR1 (EWS RNA Binding Protein 1), CCDCC117 (Coiled-Coil Domain Containing 117), KREMEN1 (Kringle Containing Transmembrane Protein 1) and ZNRF3 (Zinc And Ring Finger 3) genes, which are co-localized within a TAD located on chromosome 22 (46). The location of this SNP 5' to the $X B P 1$ gene is shown in Fig. 3b.
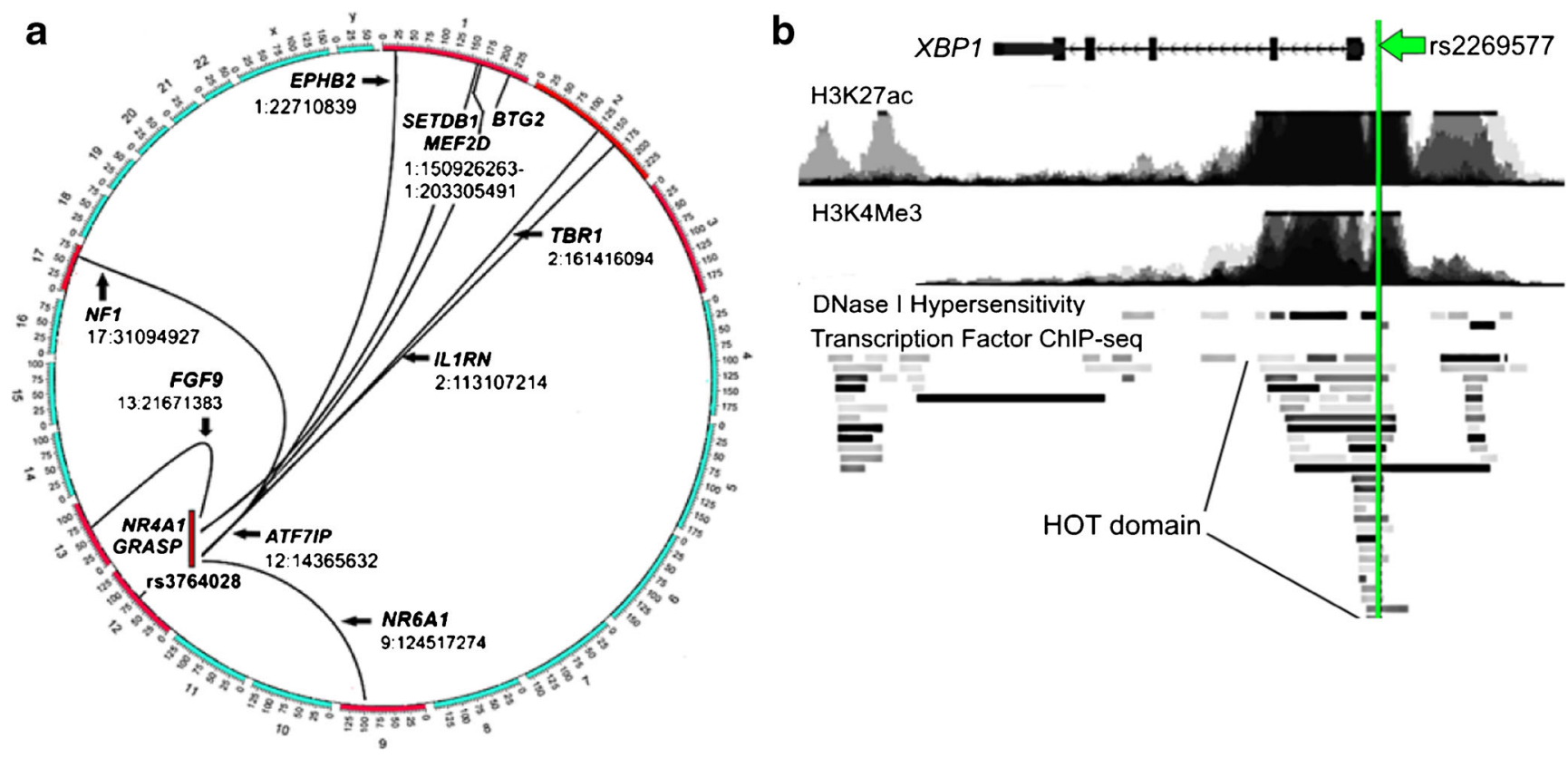

Fig. 3 Spatial context of promoters containing VPA pharmacogenomic SNPs. (a) Whole genome plot of transcriptional hub based on cis- and trans-contacts of the SNP rs3764028_G in the promoter of the GRIN2B gene based on data from Hi-C mapping of this SNP in the human neuronal cell line SK-N-SH. (b) Relative location of the VPA pharmacogenomic SNP rs2269577 (green line), with histone marks H3K27ac and H3K4me4 indicative of an active promoter, location in a DNase I hypersensitivity region indicative of open chromatin, and overlapping a HOT domain containing many TFs (39). This SNP is located within a TAD located on chromosome $22(26,600,000-28,000,000)(46)$ containing the XBPI gene as well as other genes. Inset. Screenshots from the UCSC genome browser, build $\mathrm{hg} \mid 8$, taken from the UCSC genome browser in (b) (47). 
VPA modulates a transcriptional hub consisting of 12 genes in spatial contact with the GRIN $2 B$ promoter

The 5' SNP rs3764028_G located in the distal GRIN2B promoter is in a region associated with massive chromatin reorganization in brain $(48,49)$ in rodents and human cell lines. This SNP detects a promoter that maintains spatial contact, as determined by Hi-C maps of SK-N-SH cells. SK-N-SH cells are a lymphoblastoid cell line which contains a mixture of 2 cell types. This regulatory community also contains 3 known enhancers including 2 super enhancers as well as a promoter with spatial contacts to genes associated with neuronal survival and plasticity. This replicates a result from a study in mouse brain in which researchers combined genome-wide analysis of data sets for chromatin accessibility (FAIRE-Seq) and the enhancer mark H3K27ac, in which they found a subset of genes associated with neuroprotection and plasticity that increased transcription in adult mouse brain following activation of the glutamate receptor (49). In our study, both gene set enrichment using Gene Ontology, as well as manual inspection of genes that maintain spatial contacts with the promoters and enhancers detected by our putative causal SNPs demonstrate selectivity for neuroplasticity and chromatin reorganization. In agreement with their findings, genes whose abundance increased included NR4A1, which regulates dendritic spindle density and organization in human brain following neuronal excitation, BTG2, a transcription factor that inhibits neural precursor cell proliferation and stimulates neuron cell differentiation and acts in histone arginine methylation, ILRN, which is also in contact with an enhancer associated with SNP rs2857654_A, and $\mathcal{N} F 1$, which differentially controls neural stem cell proliferation. In addition, we identified contacts with GRASP, part of a receptor complex scaffold that regulates $\mathrm{G}$ protein-coupled glutamate receptor signaling, $M E F 2 D$, which is regulated by NEUROD1, and EPHB2 (EPH Receptor B2), a developmentally-regulated receptor tyrosine kinase that functions in axonal guidance during development. This promoter also maintains long distance spatial contact with SETDB1, an H3-K9 histone methyltransferase that regulates epigenetic gene silencing to maintain stem cell pluripotency, a result that has been experimentally shown in rodent brain and cell lines by other researchers $(48,49)$.

The distal GRIN2B promoter maintains spatial interactions with genes that program neuronal cell fate in humans, and are highly responsive to VPA. These are TBR1 (T-Box, Brain 1), which is up-regulated by VPA following TBI in our animal model. TBR 1 is a TF that acts as a potent programmer of neurogenesis (50), controls the differentiation of pyramidal cells in neocortex, and controls expression of the GRIN2B gene in developing cerebral cortex (51). The other is FGF9 (Fibroblast Growth Factor 9), which is produced by developing neurons to maintain homeostasis within the surrounding milieu. It plays an important role in the regulation of embryonic development, cell proliferation, cell differentiation and cell migration, regulation of gliosis during repair and regeneration of brain tissue after damage and the differentiation and survival of neuronal cells (52). Several of these genes that exhibit spatial proximity to the GRIN2B promoter in human SK-N-SH cells are also up-regulated by VPA in the swine model of TBI and hemorrhage. These include FGF9, NR6A1, TBR1 and MEF2D.

\section{Relationship between VPA-regulated TFs and other TFs in human brain and cell types}

To understand the relationship between genes whose expression is highly regulated by VPA following brain injury in the GNS, we first examined sets of TFs which were known to be differentially regulated by VPA in brain and whose function was linked to neurogenesis and suppression of gliogenesis in human brain. Comparisons were made between VPAresponsive master TFs and their targets and other TFs in the human telencephalon, and the different sets are represented on a coronal section of the human telencephalon (Fig. 6). Adult human brain regions included the angular gyrus, anterior caudate, cingulate gyrus, hippocampal formation, inferior temporal lobe and mid-frontal lobe. Cell types included astrocytes and HUVEC, the latter a lymphoblastoid cell line derived from human vascular endothelial cells.

TFs were assigned using a subset of ENCODE brain regions and in astrocytes based on annotation of superenhancer status using histone marks such as H3K27ac and other characteristics. From this analysis, only 8 of the TFs and nuclear receptors regulated by VPA exhibit enhancer-like attributes in various brain regions. Other enriched TFs include NFIX, regulated by CHAF1B and SOX2. The correlation between VPA-TFs and TFs which function with superenhancer status in different human brain regions is significant $(p=3.12 \mathrm{E}-15)$, with this small subset accounting for $31-48 \%$ of all TFs across the telencephalon. In astrocytes, as shown in Fig. 4, although only MEF2D is represented as a VPA-TF, $17 \%$ of all TFs expressed in this cell type are regulated by VPA-TFs. HUVEC cells were used as a surrogate for CNS endothelial cells, and MEF2C, MEF2D and NR1D1 are VPA$\mathrm{TFs}$, with these $\mathrm{TFs}$ and their targets accounting for $18 \%$ of the TFs in this lymphoblastoid cell line.

Examination of the interaction and correlation between diverse datasets led to a definitive set of master TFs and their target TFs that were involved in VPA-mediated neurogenesis. Forty-seven TF targets of the 18 master transcription factors regulated by VPA include are controlled by 18 master TFs (Supplementary Table 2), but not all the master TFs are expressed in the adult human telencephalon at high abundance (Fig. 4). These data show that VPA-regulated TFs responsible for neuronal cell fate are found in the adult human 
Fig. 4 Distribution of VPAregulated master TFs and their target TFs in the VPA pathway mapped onto a coronal section of human brain. TFs in bold are VPAresponsive master TFs, which are regulated by super-enhancers according to H3K27ac marks and other attributes, based on

Supplementary Table 3 and (53).

Percentages indicate total number of VPA-regulated TFs that have been annotated as such in each region.

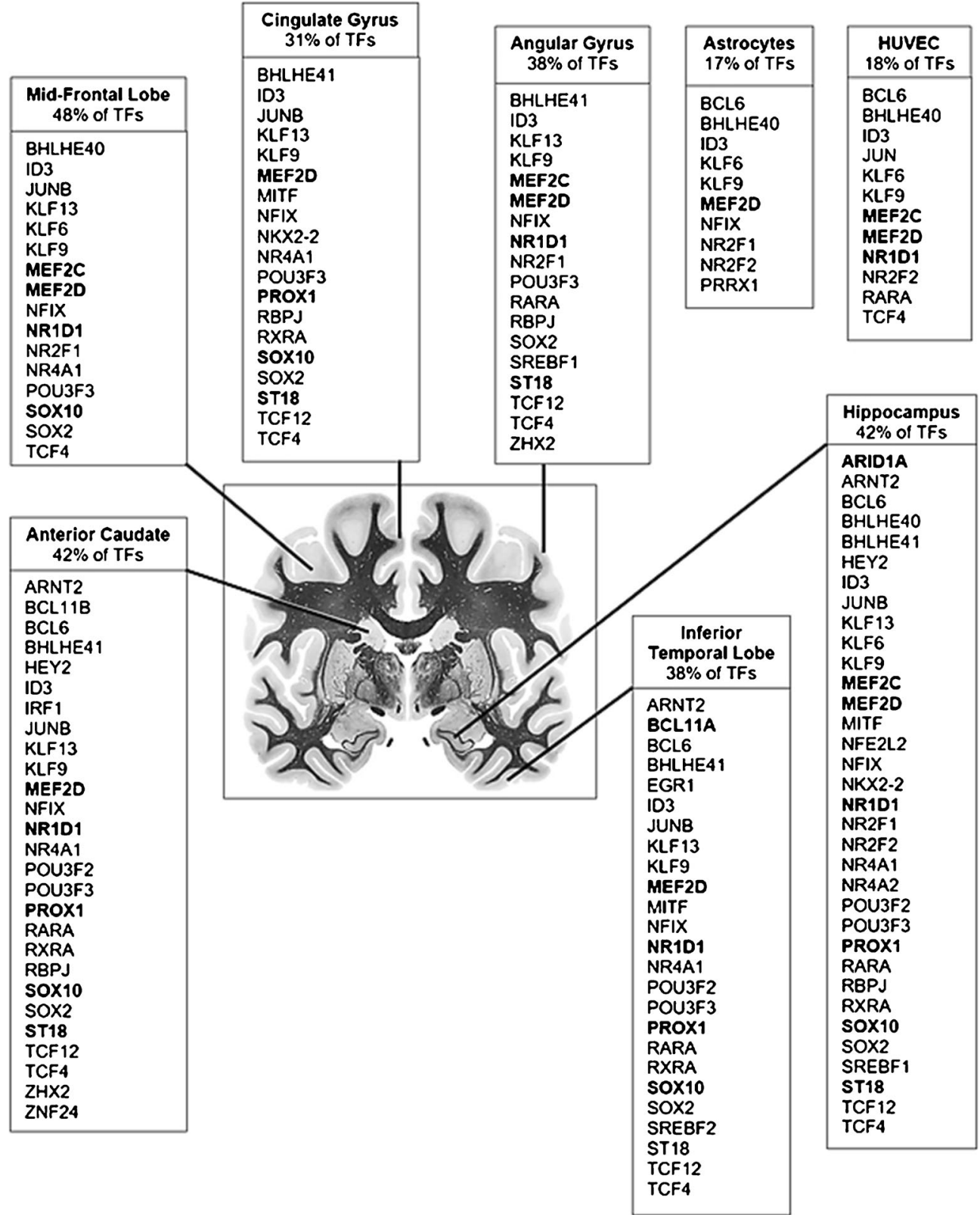

brain, and TFs that can program fibroblasts into oligodendrocytes including OLIG2 and ST18, are also present. For details concerning Fig. 4 see (53).

Next, we performed a GSEA on VPA-responsive versus non-VPA TFs found in these different adult brain regions and compared these results to those found in our animal model. Supplementary Figure 4 shows a comparison of gene set enrichment, including brain region subsets from the data used for Fig. 4, including (Supplementary Figure 4A), the set of VPA-regulated TFs and (Supplementary Figure 4B) the set of non VPA-responsive TFs. TFs found in human telencephalon that are not VPA-responsive are not enriched for neurogenesis, but are enriched for differentiation and development of other cell types.

\section{Preliminary evidence from microarray expression data from postmortem human brain tissue}

As part of a larger study examining patients with epilepsy, we obtained limited data on TF gene expression on 6 patients who were on VPA therapy at time of death (TOD) from the Human Brain Atlas of the Allen Brain Science Institute (23). These were compared to data from individuals who had no known medication history. Figure 5 shows a comparison between individuals on VPA treatment at TOD versus controls. As can be seen from this heatmap, patients on VPA had elevated levels of TFs that are responsible for neuronal cell fate specification, but reduced OLIG2 and ST18 gene expression, which are involved in the specification of glial cell fate during 


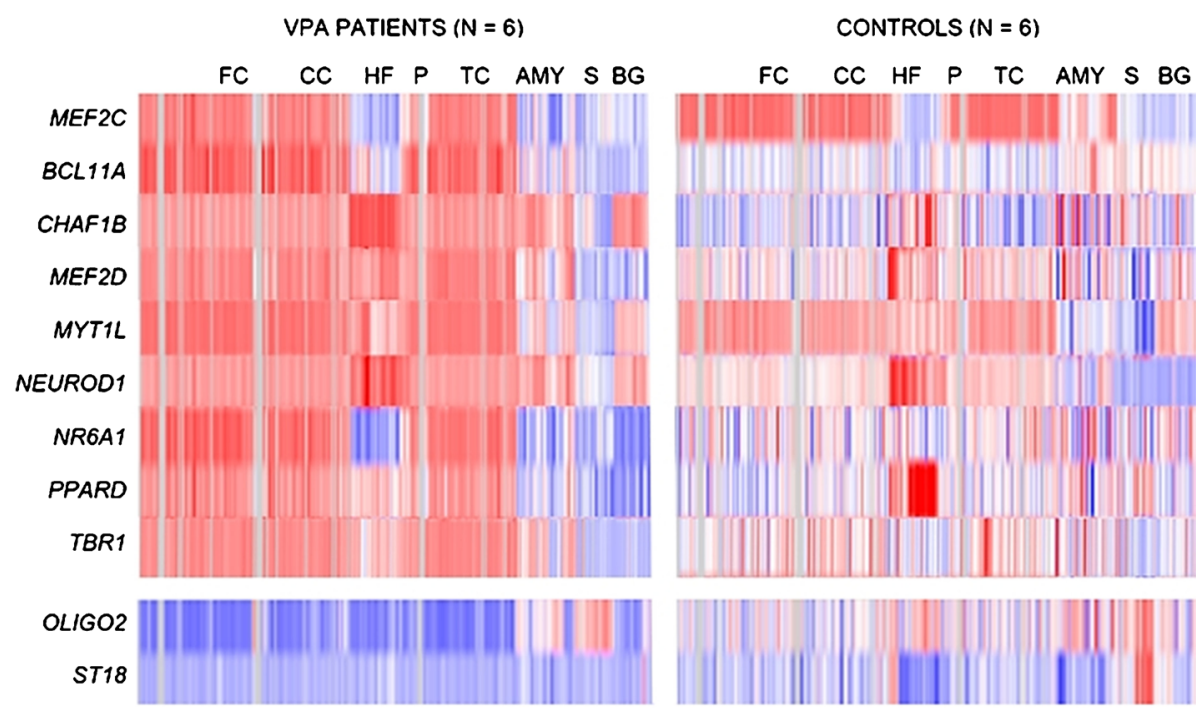

Color Map L Log2 heat map of mRNA abundance, normalized to standards (23).

Fig. 5 Differential regulation of TFs, nuclear receptor and chromatin remodeler involved in specification of cell fate in patients on VPA therapy at TOD versus controls who had no recorded history of psychotropic medication at TOD. These data obtained from a study of patients with epilepsy, and medication-free control individuals, showing samples from different regions of the telencephalon. Vertical stripes in each brain region indicates data from individual patients. AMY: Amygdala; BG: Basal ganglia; CC: Cingulate cortex; HF: Hippocampal formation; FC: Frontal cortex; P: Parahippocampal gyrus; TC: Temporal cortex; S: Septum. Courtesy, Allen Brain Science Institute (2I).

CNS development. Although $M E F 2 C$ is involved in neurogenesis and patterning of cortical structure, the heatmap shows its expression appears to be slightly less in VPA patients than in controls, especially in the amygdala. In contrast, the abundance of BCL11A, CHAF1B, MEF2D, MYT1L, NEUROD1, NR6A1, PPARD and TBR1 mRNA was significantly higher in these brain regions than in epileptic patients who were taking VPA at TOD than in control individuals $(p=4.2 \mathrm{E}-07)$.

\section{Sub-network analysis}

Analysis of VPA gene expression from expression data from the injured swine brain was made using several approaches, and several important sub-networks are shown in Fig. 6. Subsequently reconstruction of a network of the central VPA pathway in human brain that extended the animal model included genes that encode known TFs that direct cell fate in the developing CNS in the human, a subset of nuclear receptors, cytoskeletal proteins including NEFL, NEFH and INA, circadian genes, genes whose products are associated with histone acetylation, suppression of HDACs, apoptotic proteins, neurotransmitters including 15 potassium channels, corticotropin releasing hormone, cholinergic receptors, GABA receptors, opiate receptors, synaptic proteins, proteins that promoter neurite extension, axonal growth and dendritic arborization, antiangiogenic proteins, growth factors and others. Supplementary Figure 1 shows the reconstructed VPA gene regulatory network of TFs in human brain as determined by IPA ${ }^{\circledR}$ including master TFs, TFs, nuclear receptors, growth factors, selected VPA pharmacogenomic genes and related genes that are up- or down-regulated following TBA in an

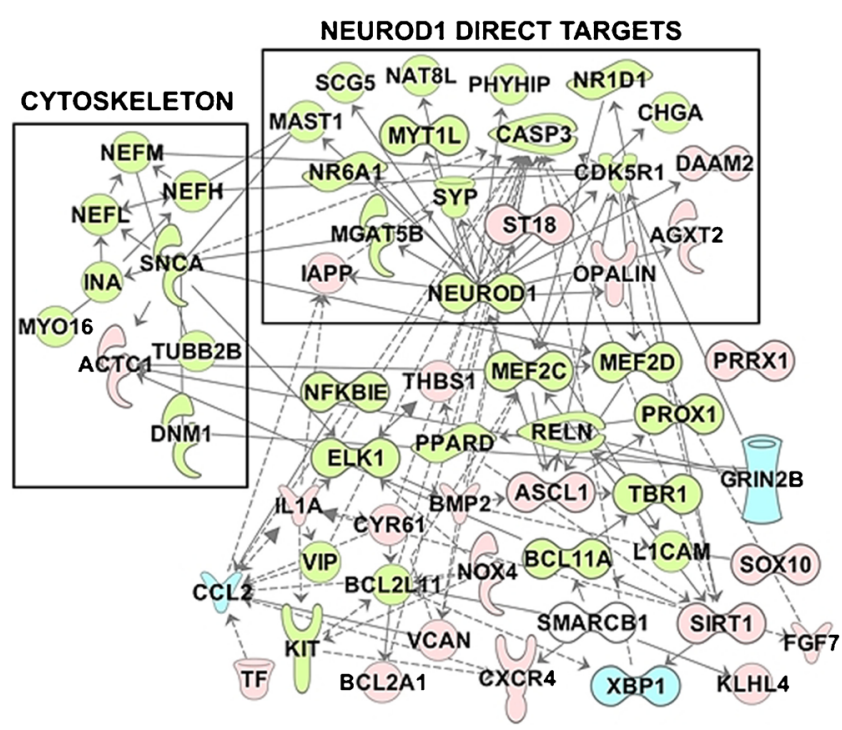

Fig. 6 Examples of subnetworks in network reconstructed from injured swine brain treated with VPA. These include 11\% of the differentiallyregulated molecules in the dataset, enriched for TFs, nuclear receptors and VPA pharmacogenomic genes (blue). Insets include a fraction of the genes associated with the cytoskeleton and nucleoplasm, and the 17 functionallyvalidated promoter targets of NEURODI. Symbols in green indicate genes up-regulated by VPA in this model and the red indicate down-regulated genes. For extension of this network to the VPA pharmacodynamic pathway in human brain, see Supplementary Figure I. In the entire dataset, the genes that discretize VPA response include CCL2, with 23 direct interactions, GRIN2B, with 20 direct interactions, and XBPI with 4 direct interactions. Pathway contacts made using the "connect" function of IPA $®(40)$. For symbol key, see Supplementary Figure I. 
animal model, as well as additional genes determined by IPA $®$ that are intimately associated with these genes in human brain using the "grow" function of the software. This forms the transcriptional foundation of the central VPA pathway in human brain. Although between 28 and 50\% of VPA-inducible master TFs are normally expressed in adult brain (Fig. 6) there are TF genes that are not normally expressed at high abundance that are responsible for activation of neurogenesis and suppression of cell fate specification of non-neuronal cell types that are upregulated following VPA therapy. The STRING database (40) leads to the same or a very similar network of TFs, as shown in Supplementary Figure 2. Following reconstruction of the network, we examined which drugs were most likely to impact this pathway based on existing data. Analysis using the IPA $®$ "upstream regulatory" toxicology analysis, the most significant $\mathrm{drug} /$ chemical regulating this network was VPA ( $p$-value 2.98E-22 using Fisher's exact test).

Supplementary Figure 1 only includes a fraction of the pathway, including direct targets such as master TFs and some of the genes they regulate, and represents a union of data from human and a swine model of TBI and hemorrhage. Green symbols show up-regulated genes following VPA treatment versus vehicle in the swine model of TBA, and red symbols are down-regulated genes following VPA treatment in the animal model of brain injury. Blue symbols are VPA pharmacogenomic genes (Table I). Symbols lacking colors are VPA-regulated genes that were predicted by IPA® using the "connect" function, as essential components of the same interconnected pathway expressed in human brain. We then compared overlap of pathway components to previously defined networks in the Reactome database (45), the IPA ${ }^{\circledR}$ database (43) and Gene Ontology (54). Previously defined biological networks across tissues and species exhibited significant overlap with the set of VPA pathway molecules. As might be expected from the widespread action of VPA-responsive TFs that direct neurogenesis, the 3 most significant overlaps were: (1) Neuronal cell fate determination in mammals ( $p$-value $<1.30 \mathrm{E}-13$; $q$-value < 4.39E-10), (2) Nuclear receptor transcription pathway in humans $(p<3.94 \mathrm{E}-13 ; q<1.67 \mathrm{E}-10)$ and $(3)$ Neurogenesis in humans $(p<5.17 \mathrm{E}-12 ; q<7.18 \mathrm{E}-10)$. Supplementary Table 2 shows a summary of the overlap of TFs found in this reconstructed VPA gene regulatory network with a matrix of known TFs responsible for: (1) GNS developmental processes, (2) Specification of germinal layers of cerebral cortex, and (3) Reprogramming/trans differentiation of different cell types to neuroblasts, neural crest, neural progenitor cells and neurotransmitter-specific neurons.

\section{Many TFs in the VPA regulatory network are located in HOT regions}

It has been found that many master TFs regulated by superenhancers are also sites of high TF occupancy (HOT regions)
(39). HOT regions correlate with decreased nucleosome density and increased nucleosome turnover and are primarily associated with open chromatin. Collectively, these HOT regions span approximately $19 \%$ of the genome in other species (39,55). Since the reconstructed VPA gene regulatory pathway contains not only master TFs normally found in adult brain (Fig. 4), and the drug induces numerous master TFs in human brain that are expressed at high abundance during GNS development, we examined the overlap between HOT regions and genes in our pathway. Many genes in the reconstructed VPA pathway were in HOT regions.

\section{DISCUSSION}

The rapid action of VPA in the adult brain appears to reduce the impact of ensuing trauma following TBI and hemorrhage, and mitigates neuronal atrophy in bipolar disorder and epilepsy. This raises the question of whether newly differentiated neurons are absorbed into active neuronal regulatory networks to become part of functional neurophysiological pathways. The highly significant changes related to neuroplasticity that is observed in our animal model of TBI and hemorrhage suggest this may be true. However, it is most probable that VPA acts through different pathways following traumatic injury than that observed in healthy tissue. Previous studies in rodent models of ischemia followed by treatment with $\mathrm{HDAC}$ inhibitors including VPA and sodium butyrate have observed activation of neurogenesis in the adult brain (10,56), although repair mechanisms may depend on the post-injury timeframe (56).

Our results show that existing sources of disparate data can be used to model transcriptional networks that are activated by VPA in the human CNS. VPA generates widespread changes in chromatin state in the genome accompanied by targeted transcriptional changes that mediate neurogenesis (12). Although VPA in combination with other small compounds may be able to induce neurogenesis (57), previous research suggests that it acts, in part, through TFs responsible for determination of neuronal cell fate, such as NEUROD1 and TBR1 ((58): Supplementary Table 3). Specific chromatin modifying protein complexes that include ARID1A are critical for chromatin remodeling during neurogenesis $(59,60)$, including acetylation of the histone mark H3K27, which defines active enhancers and promoters. Based on these findings we have devised a model that attempts to explain VPA's mechanism of action in the human adult brain (Fig. 7). This model consists of 3 components: (1) Activation of AKT1/mTOR signaling pathways by GABA receptors and/or growth factors that activate genes whose expression provides neuroprotection, (2) Opening of chromatin through HDAC inhibition, leading to histone acetylation and widespread gene expression including expression of genes involved 
Fig. 7 Putative mechanisms of VPA in human brain. (I) Activation of AKTI/mTOR signaling pathways by GABA receptors and/or growth factors that activate genes whose expression provides neuroprotection, (2) VPA acts directly to open chromatin through HDAC inhibition, leading to histone acetylation and widespread gene expression including expression of genes involved in the cell cycle, and (3) Chromatin state remodeling complexes such as npBAF containing proteins encoded by ARIDIA, BCLI IA and CHAFIB, act as intermediaries to prepare for pioneer TFs to initiate neurogenesis and repression of non-neuronal gene expression. There is evidence that PAX6 acts as a pioneer factor in concert with BAF during neurogenesis $(61,62)$. These mechanisms are not mutually exclusive

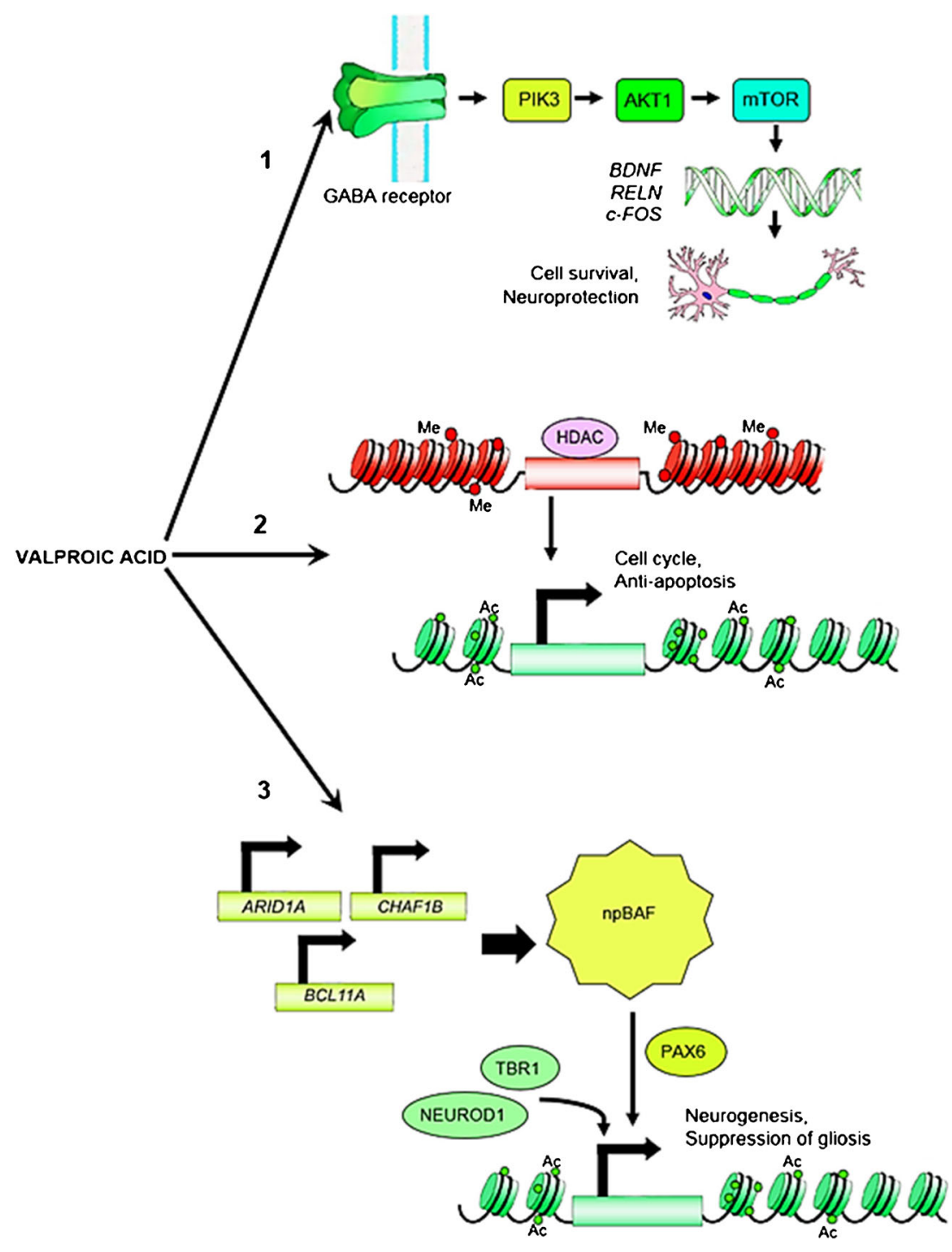

in the cell cycle, and (3) Induction of chromatin state remodeling complexes such as npBAF containing proteins encoded by ARID1A, BCL11A and CHAF1B, which act as intermediaries for pioneer $\mathrm{TF}_{\mathrm{S}}$ to initiate neurogenesis and repression of non-neuronal gene expression. These mechanisms of action are not mutual exclusive and occur concurrently with suppression of TFs such as ST18, which is responsible for glial cell fate programming during development (63).

In cell lines, treatment with VPA causes rapid and extensive chromatin decondensation, suggesting that VPA broadly and directly acetylates histones for transformation of chromatin state from heterochromatin to euchromatin. Thus, it may be that VPA acts directly to inhibit histone deacetylases. Alternatively, rapid activation of chromatin remodeling proteins such as the ARID1A component of the neural progenitors-specific chromatin remodeling complexes $\mathrm{npBAF}$ and $\mathrm{nBAF}$ may mediate chromatin remodeling following administration of VPA. Ultimately, it may be that all of these mechanisms contribute to VPA's mechanism of action in the human CNS.

Adequate data now exist to elucidate the transcriptional program that resolves all of the components that are necessary and sufficient to determine VPA's mechanism in the human brain using knowledge-based discovery. This capability has been demonstrated in other domains, including modeling of the core regulatory circuits in different human tissues and cell lines, including super-enhancers, master TFs and TFs responsible for specifying cell fate (Supplementary Tables 1, 3). In this study, we identified important master TFs such as NEUROD1 and TBR 1 that are induced by VPA following TBI in adult brain, but normally expressed at low levels in the mature human telencephalon.

Preliminary data from postmortem human brain must be considered preliminary, as complete medical histories are not available on either patients or controls, there is heterogeneity in the data, and this microarray expression reflects variability 
associated with a single time point in the analysis of postmortem human brain tissue lacking cause of death in most cases. In addition, although controls were selected based on lack of medication history, medical history was not well characterized.

Since VPA relieves symptoms in disorders such as bipolar disorder, which involves neuronal loss and neural atrophy (64), it is tempting to speculate that VPA may have a positive effect on a range of damaged brain tissue, from mild atrophy to the critical damage that is often observed following TBI. Conversely, VPA may generate aberrant hyperplasia of different cell types in human brain as has been suggested (65). This could account for rebound seizures and CNS impairment including encephalopathy after chronic treatment with VPA (66).

Further investigation is warranted to completely understand how VPA and other HDAC inhibitors can provide functional recovery after severe trauma to the human brain and spinal cord, and to a lesser extent, suppress epileptogenesis, alleviate mania in bipolar disorder, and act as an analgesic in migraine. Modeling using rich data resources as was performed in the current study generate testable hypotheses for more focused experiments in subsequent studies.

Open Access This article is distributed under the terms of the Creative Commons Attribution 4.0 International License (http://creativecommons.org/licenses/by/4.0/), which permits unrestricted use, distribution, and reproduction in any medium, provided you give appropriate credit to the original author(s) and the source, provide a link to the Creative Commons license, and indicate if changes were made.

\section{REFERENCES}

1. Ghodke-Puranik Y, Thorn CF, Lamba JK, et al. Valproic acid pathway: pharmacokinetics and pharmacodynamics. Pharmacogenet Genomics. 2013;23(4):236.

2. Perucca E. Pharmacological and therapeutic properties of valproate. CNS Drugs. 2002;16(10):695-714.

3. Göttlicher M, Minucci S, Zhu P, et al. Valproic acid defines a novel class of HDAC inhibitors inducing differentiation of transformed cells. EMBO J. 2001;20(24):6969-78.

4. Gurvich N, Tsygankova OM, Meinkoth JL, Klein PS. Histone deacetylase is a target of valproic acid-mediated cellular differentiation. Cancer Res. 2004;64(3):1079-86.

5. Halaweish I, Bambakidis T, Chang Z, et al. Addition of low-dose valproic acid to saline resuscitation provides neuroprotection and improves long-term outcomes in a large animal model of combined traumatic brain injury and hemorrhagic shock. J Trauma Acute Care Surg. 2015;79(6):911-9.

6. Dash PK, Orsi SA, Zhang M, et al. Valproate administered after traumatic brain injury provides neuroprotection and improves cognitive function in rats. PLoS One. 2010;5(6), el 1383.

7. Lv L, Sun Y, Han X, Xu CG, Tang YP, Dong Q. Valproic acid improves outcome after rodent spinal cord injury: potential roles of histone deacetylase inhibition. Brain Res. 2011;1396:60-8.
8. Guo Z, Zhang L, Wu Z, Chen Y, Wang F, Chen G. In vivo direct reprogramming of reactive glial cells into functional neurons after brain injury and in an Alzheimer's disease model. Cell Stem Cell. 2014;14(2):188-202.

9. Dekker SE, Bambakidis T, Sillesen M, et al. Effect of pharmacologic resuscitation on the brain gene expression profiles in a swine model of traumatic brain injury and hemorrhage. J Trauma Acute Care Surg. 2014;77(6):906-12.

10. Kim HJ, Leeds P, Chuang DM. The HDAC inhibitor, sodium butyrate, stimulates neurogenesis in the ischemic brain. $\mathrm{J}$ Neurochem. 2009;110(4):1226-40.

11. Halsall JA, Turan N, Wiersma M, Turner BM. Cells adapt to the epigenomic disruption caused by histone deacetylase inhibitors through a coordinated, chromatin-mediated transcriptional response. Epigenetics Chromatin. 2015;8(1):1.

12. Yi J, Zhang L, Tang B, et al. Sodium valproate alleviates neurodegeneration in SCA3/MJD via suppressing apoptosis and rescuing the hypoacetylation levels of histone $\mathrm{H} 3$ and H4. PLoS One. 2013;8(1), e54792.

13. Marchion DC, Bicaku E, Daud AI, Sullivan DM, Munster PN. Valproic acid alters chromatin structure by regulation of chromatin modulation proteins. Cancer Res. 2005;65(9):3815-22.

14. Kelkhoff D, Downing T, Li S. Mechanotransduction to epigenetic remodeling. In: Molecular and cellular mechanobiology. New York: Springer; 2016. p. 163-173.

15. Kundaje A, Meuleman W, Ernst J, et al. Integrative analysis of 111 reference human epigenomes. Nature. 2015;518(7539):317-30.

16. Rao SS, Huntley MH, Durand NC, et al. A 3D map of the human genome at kilobase resolution reveals principles of chromatin looping. Cell. 2014;159(7):1665-80.

17. Gonzalez-Sandoval A, Gasser SM. On TADs and LADs: Spatial Control Over Gene Expression. Trends Genet. 2016;32(8):485-95.

18. Lupiáñez DG, Kraft K, Heinrich V, et al. Disruptions of topological chromatin domains cause pathogenic rewiring of gene-enhancer interactions. Cell. 2015;161(5):1012-25.

19. Lee D, Gorkin DU, Baker M, et al. A method to predict the impact of regulatory variants from DNA sequence. Nat Genet. 2015;47(8): 955-61.

20. Ramani V, Shendure J, Duan Z. Understanding Spatial Genome Organization: Methods and Insights. Genomics Proteomics Bioinformatics. 2016;14(1):7-20.

21. Sunkin SM, Ng L, Lau C, et al. Allen Brain Atlas: an integrated spatio-temporal portal for exploring the central nervous system. Nucleic Acids Res. 2013;41(D1):D996-1008.

22. Higgins GA, Allyn-Feuer A, Athey BD. Epigenomic mapping and effect sizes of noncoding variants associated with psychotropic drug response. Pharmacogenomics. 2015;16(14):1565-83.

23. Kulkarni AV, Aziz B, Shams I, Busse JW. Comparisons of citations in Web of Science, Scopus, and Google Scholar for articles published in general medical journals. JAMA. 2009;302(10):1092-6.

24. Jessen WJ, Landschulz KT, Turi TG, Reams RY. Mining PubMed for biomarker-disease associations to guide discovery. PeerJ. 2015;3, e1802.

25. Zarin DA, Tse T, Williams RJ, Califf RM, Ide NC. The ClinicalTrials. gov results database-update and key issues. N Engl J Med. 2011;364(9):852-60. DrugBank database, version, 2 (2015)

26. Kaplun A, Hogan JD, Schacherer F, et al. PGMD: a comprehensive manually curated pharmacogenomic database. The Pharmacogenomics J. 2016;16(2):124-8.

27. Dawson HD. A comparative assessment of the pig, mouse and human genomes. The minipig in biomedical research. 2011;323-42.

28. Jones B. Evolutionary genetics: Becoming human: Identifying human accelerated regulatory DNA. Nat Rev Genet. 2015;16(8): 438-9. 
29. Boley N, Wan KH, Bickel PJ, Celniker SE. Navigating and mining modENCODE data. Methods. 2014;68(1):38-47.

30. di Iulio J, Bartha I, Wong E, Yu HC, Hicks M, Shah N, et al. The human functional genome defined by genetic diversity. bioRxiv. $2016: 082362$

31. He X, Li Y, Liu Z, et al. The association between CCL2 polymorphisms and drug-resistant epilepsy in Chinese children. Epileptic Disord. 2013;15(3):272-7.

32. Hung CC, Ho JL, Chang WL, et al. Association of genetic variants in six candidate genes with valproic acid therapy optimization. Pharmacogenomics. 2011;12(8):1107-17.

33. Kim B, Kim CY, Lee MJ, Joo YH. Preliminary evidence on the association between XBP1-116C/G polymorphism and response to prophylactic treatment with valproate in bipolar disorders. Psychiatry Res. 2009;168(3):209-12.

34. Rackham OJ, Firas J, Fang H, Oates ME, Holmes ML, Knaupp $\mathrm{AS}$, et al. A predictive computational framework for direct reprogramming between human cell types. Nat Genet. 2016;48: 331-35. doi:10.1038/ng.3487

35. Gopalakrishnan S, Hor P, Ichida JK. New approaches for direct conversion of patient fibroblasts into neural cells. Brain Res. 2017;1656:2-13

36. Neph S, Stergachis AB, Reynolds A, Sandstrom R, Borenstein E, Stamatoyannopoulos JA. Circuitry and dynamics of human transcription factor regulatory networks. Cell. 2012;150(6):1274-86.

37. Teng L, He B, Wang J, Tan K. 4DGenome: a comprehensive database of chromatin interactions. Bioinformatics. 2015;31(15): 2560-4.

38. Saint-André V, Federation AJ, Lin CY, et al. Models of human core transcriptional regulatory circuitries. Genome Res. 2016;26(3): 385-96.

39. Li H, Liu F, Ren C, Bo X, Shu W. Genome-wide identification and characterization of HOT regions in the human genome. bioRxiv. 2016;036152.

40. Szklarczyk D, Franceschini A, Wyder S, Forslund K, Heller D, Huerta-Cepas J, et al. STRING v10: protein-protein interaction networks, integrated over the tree of life. Nucleic Acids Res. 2014;gku1003.

41. Langfelder P, Horvath S. WGCNA: an R package for weighted correlation network analysis. BMC Bioinform. 2008;9(1):1.

42. Kestler HA, Wawra C, Kracher B, Kühl M. Network modeling of signal transduction: establishing the global view. Bioessays. 2008;30(11-12):1110-25.

43. Krämer A, Green J, Pollard J, Tugendreich S. Causal analysis approaches in ingenuity pathway analysis (ipa). Bioinformatics. 2013;btt703.

44. Cerami EG, Gross BE, Demir E, et al. Pathway Commons, a web resource for biological pathway data. Nucleic Acids Res. 2011;39 suppl 1:D685-90.

45. Croft D, Mundo AF, Haw R, et al. The Reactome pathway knowledgebase. Nucleic Acids Res. 2014;42(D1):D472-7.

46. Wang $\mathrm{S}, \mathrm{Su} \mathrm{JH}$, Beliveau BJ, et al. Spatial organization of chromatin domains and compartments in single chromosomes. Science. 2016;353(6299):598-602.

47. Speir ML, Zweig AS, Rosenbloom KR, et al. The UCSC Genome Browser database: 2016 update. Nucleic Acids Res. 2016;44(D1): D717-25.

48. Thakurela S, Sahu SK, Garding A, Tiwari VK. Dynamics and function of distal regulatory elements during neurogenesis and neuroplasticity. Genome Res. 2015;25(9):1309-24.
49. Bharadwaj R, Peter CJ, Jiang Y, et al. Conserved higher-order chromatin regulates NMDA receptor gene expression and cognition. Neuron. 2014;84(5):997-1008.

50. Mihalas AB, Hevner RF. Control of neuronal development by TBox genes in the brain. Curr Top Dev Biol. 2016;122(2017):279312

51. Notwell JH, Heavner WE, Darbandi SF, et al. TBRl regulates autism risk genes in the developing neocortex. Genome Res. 2016;26(8):1013-22.

52. Hadjab S, Franck MC, Wang Y, et al. A local source of FGF initiates development of the unmyelinated lineage of sensory neurons. J Neurosci. 2013;33(45):17656-66.

53. Berto S, Perdomo-Sabogal A, Gerighausen D, Qin J, Nowick K. A consensus network of gene regulatory factors in the human frontal lobe. Front Genet. 2016;7.

54. Mi H, Poudel S, Muruganujan A, Casagrande JT, Thomas PD. PANTHER version 10: expanded protein families and functions, and analysis tools. Nucleic Acids Res. 2016;44(D1):D336-42.

55. Roy S, Ernst J, Kharchenko PV, et al. Identification of functional elements and regulatory circuits by Drosophila modENCODE. Science. 2010;330(6012):1787-97.

56. Liu XS, Chopp M, Kassis H, et al. Valproic acid increases white matter repair and neurogenesis after stroke. Neuroscience. 2012:220:313-21

57. Chu $\mathrm{T}$, Zhou $\mathrm{H}$, Wang $\mathrm{T}$, et al. In vitro characteristics of valproic acid and all-trans-retinoic acid and their combined use in promoting neuronal differentiation while suppressing astrocytic differentiation in neural stem cells. Brain Res. 2015;1596:31-47.

58. Vierbuchen T, Ostermeier A, Pang ZP, Kokubu Y, Südhof TC, Wernig M. Direct conversion of fibroblasts to functional neurons by defined factors. Nature. 2010;463(7284):1035-41.

59. Greyghton MP, Cheng AW, Welstead GG, et al. Histone H3K27ac separates active from poised enhancers and predicts developmental state. Proc Natl Acad Sci. 2010;107(50):21931-6.

60. Son EY, Crabtree GR. The role of BAF (mSWI/SNF) complexes in mammalian neural development. In: American Journal of Medical Genetics Part C: Seminars in Medical Genetics. 2014;166(3):333-49.

61. Manuel MN, Mi D, Mason JO, Price DJ. Regulation of cerebral cortical neurogenesis by the Pax6 transcription factor. Front Cell Neurosci. 2015;9:70.

62. Ninkovic J, Steiner-Mezzadri A, Jawerka M, et al. The BAF complex interacts with Pax6 in adult neural progenitors to establish a neurogenic cross-regulatory transcriptional network. Cell Stem Cell. 2013;13(4):403-18.

63. Tesar, Paul J., Robert H. Miller, Fadi J. Najm. Cell fate conversion of differentiated somatic cells into glial cells. U.S. Patent Application 13/280,562, filed April 26, 2012.

64. Phillips ML, Swartz HA. A critical appraisal of neuroimaging studies of bipolar disorder: toward a new conceptualization of underlying neural circuitry and a road map for future research. Am J Psychiatry. 2014;171(8):829-43.

65. Schurr J, Coras R, Rössler K, Pieper T, Kudernatsch M, Holthausen $\mathrm{H}$, et al. Mild Malformation of cortical development with oligodendroglial hyperplasia in frontal lobe epilepsy: a new clinico-pathological entity. Brain Pathol. 2017;27:26-35.

66. Guida M, Iudice A, Bonanni E, Giorgi FS. Effects of antiepileptic drugs on interictal epileptiform discharges in focal epilepsies: an update on current evidence. Expert Rev Neurother. 2015;15(8): 947-59. 